\title{
Quasi-one dimensional fluids that exhibit higher dimensional behavior
}

\author{
SILVINA M. GATICA* \\ Department of Physics and Astronomy, \\ Howard University, \\ Washington, DC, 20059, USA \\ sgatica@howard.edu \\ M. MERCEDES CALBI \\ Department of Physics, Southern Illinois University, Carbondale, IL USA \\ GEORGE STAN \\ Department of Chemistry, University of Cincinnati, Cincinnati, OH 45221, USA \\ R. ANDREEA TRASCA \\ Dieffenbachstr. 58A, 10967 Berlin, Germany \\ MILTON W. COLE* \\ Department of Physics, \\ Penn State University, \\ University Park, PA 16802, USA \\ miltoncole@aol.com
}

\begin{abstract}
Fluids confined within narrow channels exhibit a variety of phases and phase transitions associated with their reduced dimensionality. In this review paper, we illustrate the crossover from quasi-one dimensional to higher effective dimensionality behavior of fluids adsorbed within different carbon nanotubes geometries. In the single nanotube geometry, no phase transitions can occur at finite temperature. Instead, we identify a crossover from a quasi-one dimensional to a two dimensional behavior of the adsorbate. In bundles of nanotubes, phase transitions at finite temperature arise from the transverse coupling of interactions between channels.
\end{abstract}

\footnotetext{
* Corresponding author
} 


\section{INTRODUCTION}

One of the most interesting topics within modern condensed matter physics is that of phenomena in reduced dimensionality, resulting from some degree of spatial localization of the particles comprising a system. [1] For example, chemists, materials scientists and physicists have created and explored numerous physical systems in which atoms and molecules are confined within quasi-one dimensional (Q1D) environments. The variety of these systems is remarkable, such as the peapod geometry, i.e., a line of buckyballs within a carbon nanotube, [2, 3] fluids within artificial materials created by templating [4, 5] and Q1D optical lattices created by laser fields. (7] Unfortunately, as far as we know, there exists no comprehensive review of this general problem, although many relevant subfields have been summarized. [8, 9, 10, 11] The present paper addresses a small subset of this exciting research field. Specifically, we consider problems involving fluids, both classical and quantum, confined within Q1D channels, the focus of our group's research during the last decade. 12]

Here, the term Q1D refers to a system in which particles move in an external potential field $V(\mathbf{r})$ which is either constant or slowly varying in one direction $(z)$, while $V(\mathbf{r})$ is strongly localizing in the two other (transverse) directions. In the case of quantum particles, for which the transverse spectrum is discrete, one expects that the corresponding degrees of freedom are frozen out at low temperature $(T)$; transverse excitation does occur at higher $T$, as determined by the gaps in the transverse spectrum of states. This plausible expectation is borne out in some cases, but we shall see that there can be dramatic consequences of the transverse degrees of freedom in other cases, even at low $T$.

One of the many exciting aspects of strictly 1D physics is its susceptibility to weak perturbations. The reason for this behavior arises from the fact that (in all practical situations) [13] no phase transition can exist in a purely 1D system at any finite $T$, even though the ground state may exhibit symmetry-breaking order. Thus, there do exist transitions at $T$ identically equal to zero. A familiar example is the 1D Ising model, for which the correlation length and susceptibility both diverge as $T$ approaches 0 .

Since the purely 1D system has no finite $T$ transition, what brings about more interesting behavior in the Q1D case? As discussed below, the difference can arise from considering a set of parallel 1D systems which are weakly coupled. Alternatively, the behavior can happen because the system is only $1 \mathrm{D}$ insofar as the transverse dimensions are finite, unlike the length in the $\mathrm{z}$ direction, $\mathrm{L}$, which achieves the thermodynamic limit.....but the transverse dimensions are large enough to have an observable effect (e.g. low energy gaps). A third, more surprising, origin of interesting phenomena is when the system is a collection of noninteracting 1D systems, with "quenched" heterogeneity, which are coupled to a particle bath, so they possess a common chemical potential.

The next section discusses the conceptually and computationally simplest case of a Q1D system: a low density, noninteracting gas within a single channel; then, the solution of the one-particle Schrödinger equation determines the physical behavior. Section 3 considers the case of many interacting particles within a single channel; such a problem is often used as a model for fluids within regular or irregular porous materials. Section 4 considers the problem of Q1D channels containing fluids that interact with one another as well as with fluids occupying other channels. 


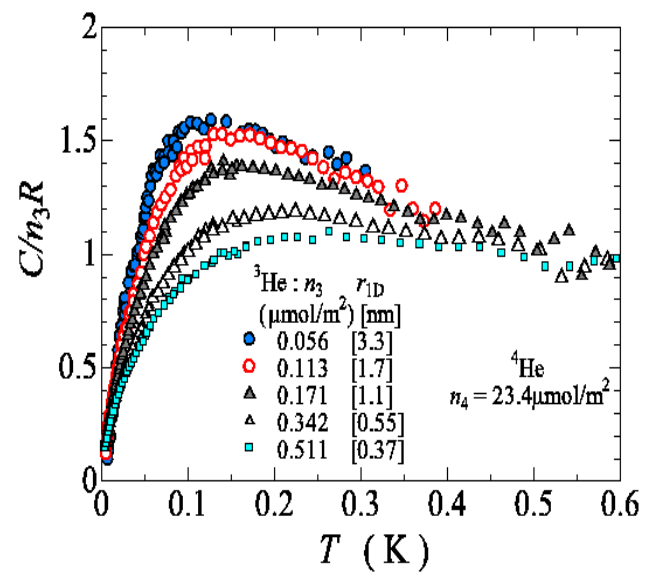

(a)

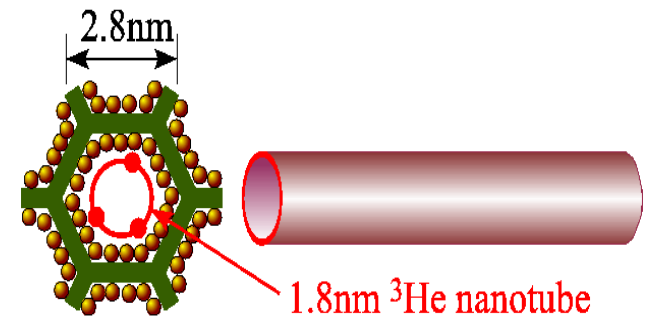

(b)

FIG. 1: Left: Experimental heat capacity for ${ }^{3} \mathrm{He}$ at various densities, inside ${ }^{4}$ He-coated FSM-16, from Ref. [5]. Right: schematic depiction of the geometry, from Ref. [6]. The tube represents the environment experienced by a ${ }^{3} \mathrm{He}$ atom.

\section{LOW DENSITY GAS IN A SINGLE Q1D CHANNEL}

Our first problem is conceptually and calculationally simple: a low density gas confined within a Q1D geometry; nevertheless, it provides interesting, and sometimes surprising, results. One example that has been studied extensively is that of a gas inside single carbon nanotubes, e.g. quantum fluids or the "peapod" case of $\mathrm{C}_{60}$ molecules. [3] Another is the case of quantum gases inside templated regular pores. [5] A third example is the so-called groove region between two nanotubes, e.g. on the outside of a bundle of nanotubes. [14, 15, 16]

A classical noninteracting gas has a kinetic energy per particle of $(3 / 2) k_{B} T$ and a mean potential energy $\langle U\rangle$ determined by its interaction with the environment. For the case of a particle localized near the $\mathrm{z}$ axis within a channel, $\langle U\rangle=k_{B} T$, due to two transverse directions of excitation. Hence, the classical specific heat per particle is $[C(T) / N]_{\text {classical }}=$ $(5 / 2) k_{B}$. For a quantum gas, instead, the transverse degrees of freedom are frozen out at low $T$, so one expects $[C(T) / N]_{\text {quantum }}=(1 / 2) k_{B}$. The generalization to $D$ "effective" dimensions yields this expression for the dimensionless specific heat, $C^{*}$, of a noninteracting Boltzmann gas:

$$
C * \equiv C /\left(N k_{B}\right)=D / 2
$$

Fig. 1 shows experimental results for $C^{*}$ in the case of ${ }^{3} \mathrm{He}$ inside of FSM-16, a material consisting of straight hexagonal pores of cross-sectional distance of order 2 to $3 \mathrm{~nm}$, precoated with a thin film of ${ }^{4} \mathrm{He}$. At the low densities shown here, the ${ }^{3} \mathrm{He}$ gas can be assumed to be noninteracting, although interaction effects appear at higher density. [17] The behavior observed inside FSM-16 can be understood by analogy to calculations in Fig. 2, for a noninteracting gas of ${ }^{4} \mathrm{He}$ within a carbon nanotube. Note the overall similarity of these two figures.

In Fig. 2, 18] one observes that the low $T$ limit is $C^{*}=1 / 2$, corresponding to a $1 \mathrm{D}$ classical gas, as expected. At high $T$, instead, the limit is $C^{*}=1$; this limit is interpreted as that of a $2 \mathrm{D}$ gas moving on the inner surface of the nanotube. The bump at intermediate 


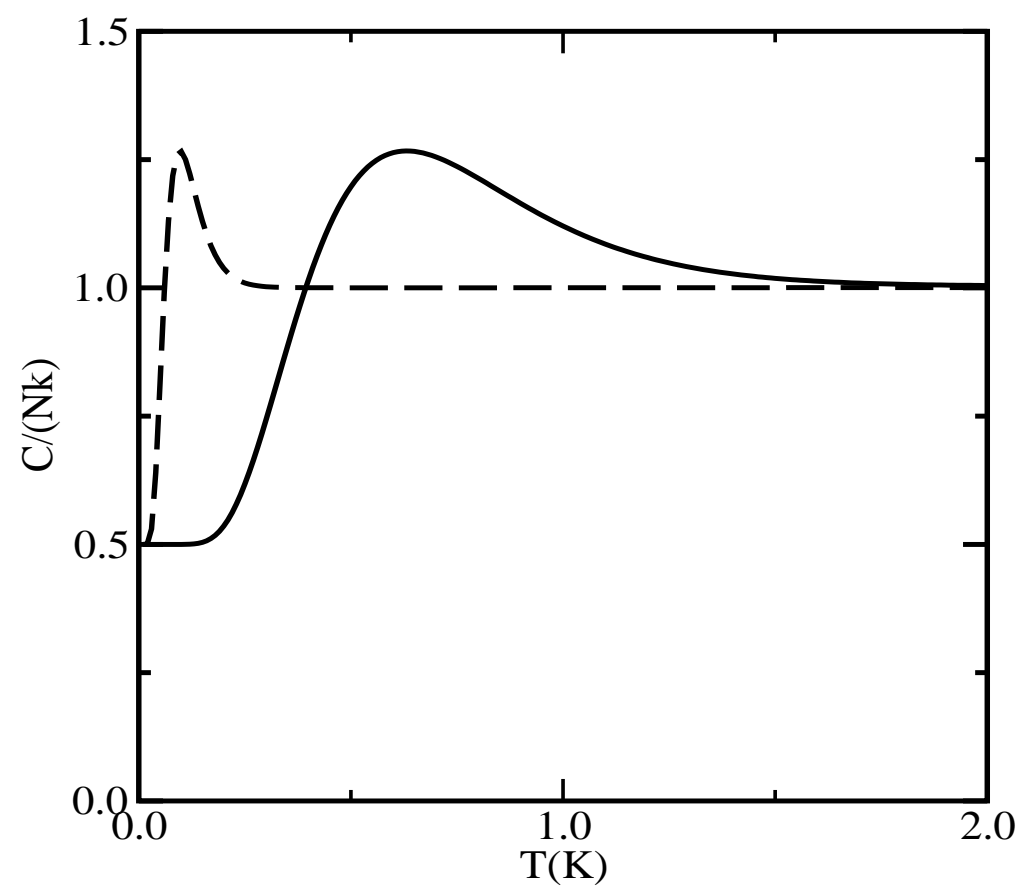

FIG. 2: Heat capacity for a low density gas of ${ }^{4} \mathrm{He}$ atoms within a single wall carbon nanotube, for cases $R=0.8 \mathrm{~nm}$ (dashes) and $0.5 \mathrm{~nm}$ (full curve). Quantum statistical effects are omitted from the calculation. Adapted from Ref. [18].

$T$ is a general property found for spectra, such as that of the rigid rotor, [19] for which the inter-level spacing increases with quantum number. In the present case, the relevant spectrum is that arising from the azimuthal kinetic energy,

$$
E_{\theta}=(\hbar \nu)^{2} /\left(2 m\langle\rho\rangle^{2}\right) \equiv \nu^{2} k_{B} \Theta
$$

Here $\nu=0, \pm 1, \pm 2, \ldots$ is the azimuthal quantum number and $\rho$ is the radial coordinate, while $\Theta$ is defined as a temperature characteristic of azimuthal excitation. The peak in the specific heat occurs at a temperature near $3 \Theta$ for both radii considered in Fig. 2, so its position serves as a benchmark from which one can determine the value of $\langle\rho\rangle$. Note that inside a nanotube, the relevant value of $\langle\rho\rangle$ is typically $R-\sigma$, where $\sigma$ is the gas-surface hard core interaction length. The key qualitative difference between the behaviors seen in Figs. 1 and 2 is that the experimental data in Fig. 1 plunge to $C^{*}=0$ as $T \rightarrow 0$. This is an effect of quantum degeneracy, manifested in Nernst's law, as found in explicit calculations which revise Fig. 2 by taking quantum statistics into account. [20]

There have been many theoretical studies of gas adsorption in the presence of nanotubes. [8, 9, 10, 11, 12] In most treatments of these systems, one assumes that neighboring tubes are parallel. In that case, there exists a region of space- the so-called "groove"which is a 1D channel with a strongly attractive potential, created by the adjacent tubes. The adsorbed gas then exhibits Q1D behavior at low $N$. However, one can also inquire about the case when the tubes are not quite parallel, but instead diverge, leaving a particularly attractive region between them, with a minimum potential energy $\left(V_{0}\right)$ located at equilibrium position $r_{0}$. In a forthcoming study, [1] we will report remarkable results for this 
geometry, as exemplified in Fig. 3. The low $T$ behavior is that of a gas in a $1 \mathrm{D}$ harmonic potential, $V \simeq V_{0}+k_{z}\left(z-z_{0}\right)^{2} / 2$, where $k_{z}$ is the force constant for particle motion parallel to the $z$ axis, midway between and nearly parallel to the tubes' axes. We introduce the characteristic temperature for this motion, $T_{z} \equiv \hbar\left(k_{z} / m\right)^{1 / 2} / k_{B}$ and a reduced temperature $T^{*} \equiv T / T_{z}$. For $T^{*}<<1$, the specific heat is of Arrhenius form, as seen in the figure, while for $T^{*} \sim 1, C^{*} \sim 1$, the specific heat of a 2D gas. That behavior might not have been anticipated, at first glance, because for small divergence half-angle $\gamma$, one might have expected 1D behavior, i.e., $C^{*}=1 / 2$. Another surprise is the high $T^{*}$ limiting behavior, $T^{*} \rightarrow 7 / 4$. This peculiar result arises because the relevant particles' motions are those in the plane perpendicular to the $x-z$ plane of the nanotubes, for which the potential variation is unusual- proportional to $y^{4}$.

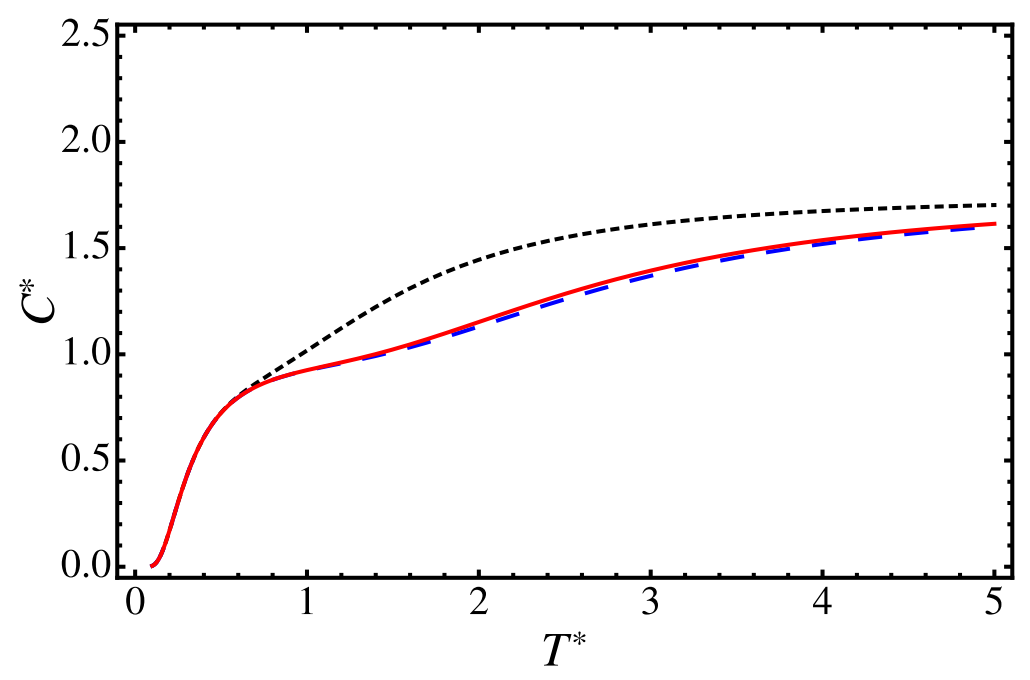

FIG. 3: Reduced specific heat $\mathrm{C}^{*}$ for the gases $\mathrm{He}$ (dashed-blue), $\mathrm{H}_{2}$ (solid-red) and Ne (shortdashed-black) between two nearly parallel nanotubes, each with $R=0.7 \mathrm{~nm}$, and a divergence half-angle $\gamma=0.5$ degrees. From Ref. [1].

\section{MANY INTERACTING PARTICLES IN A CHANNEL OF FINITE WIDTH}

From the perspective of phase transitions, a channel of finite width is a Q1D system, so that no true thermodynamic singularities can occur. This means that many attempts to explore behavior in porous media with single channel models (such as cylindrical and slit pores) cannot accurately describe phase transition behavior that is seen in genuinely 3D porous media. Nevertheless, these models may provide good semiqualitative predictive power, sufficient for most purposes since the fully 3D geometry is not known. We have explored a variety of such models, with several different goals. These include assessing the accuracy of simplifying models, such as mean-field-theory (MFT) and the use of periodic boundary conditions. Both of these approximations are suspect, at first glance, due to the important role of fluctuations in 1D statistical physics. 


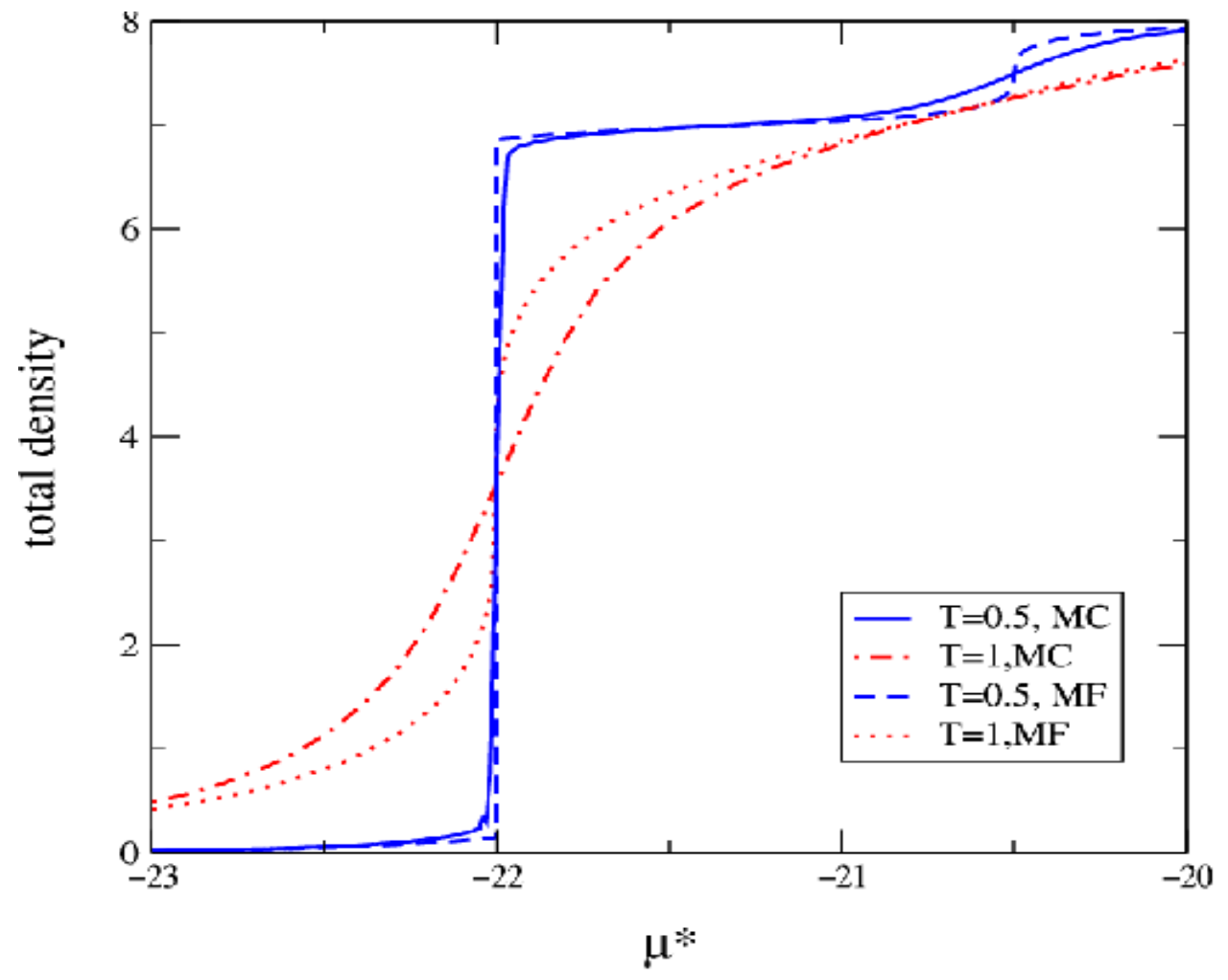

FIG. 4: Mean field isotherms compared with exact Monte Carlo isotherms as a function of reduced chemical potential and temperature, from Ref. [21].

Fig. 4 presents adsorption isotherms for uptake in a single cylindrical pore, described by a lattice gas model.21] In this model, continuous space is discretized into adsorption sites, which may be either occupied or empty in any microstate of the system. In the specific model used here, the set of sites (within one transverse section) consists of one axial site and seven "cylindrical shell" sites, corresponding to positions near the inner boundary of the nanotube. The ensemble of sites include an infinite sequence of such layers of eight sites. The energy of the system includes interactions between particles occupying these sites, plus interactions between particles and the substrate host.

Fig. 4 compares (numerically) exact results with those obtained from MFT. The results are quite similar, overall, given the highly expanded scale of reduced chemical potential $\left(\mu^{*}\right)$. Note that the spurious transition seen in MFT (at reduced temperature $T=0.5, \mu^{*}=-22$ ) is not very different from the nearly discontinuous isotherm seen in the exact results.

\section{REAL TRANSITIONS OF GASES WITHIN WEAKLY COUPLED Q1D CHANNELS}

Consider a geometry consisting of a set of parallel Q1D fluids, as in gases within or between nanotubes comprising a bundle of such tubes. While no transition can occur for an isolated Q1D system, once coupling between such systems is present, a finite temperature 


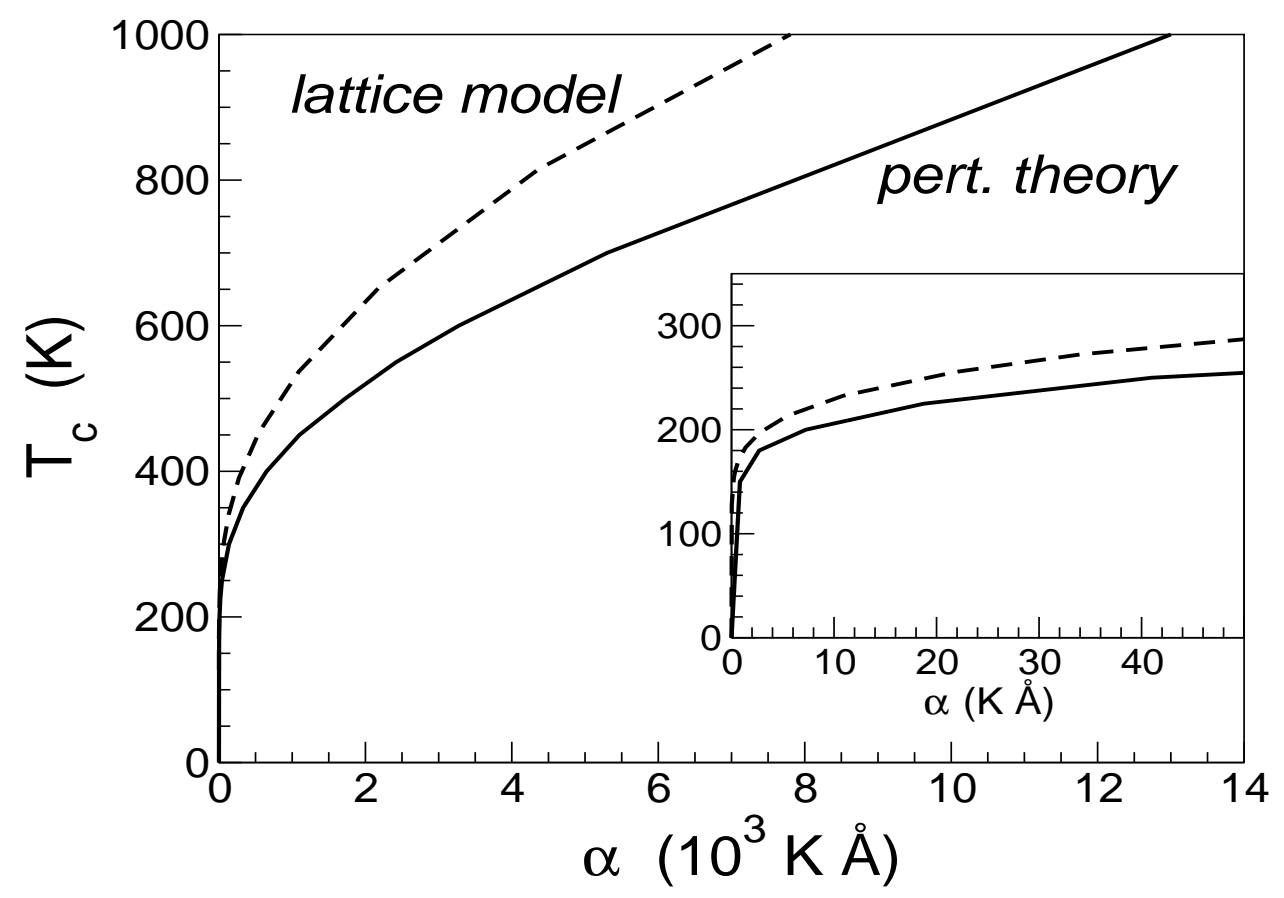

FIG. 5: Critical temperature $T_{c}$ as a function of the transverse interaction. Perturbation theory results and predictions from the anisotropic lattice gas model are compared. From Ref. [3].

transition can occur. Fig. 5 presents results for such a geometry. [3] The critical temperature $T_{c}$ is shown as a function of the transverse coupling $\alpha$, for the case of buckyballs confined within parallel nanotubes. As indicated, rather similar results were obtained from exact solutions for a lattice-gas model and from a perturbation theory. In the latter case, the unperturbed equation of state was the exact 1D result for a model with Lennard-Jones interactions between the buckyballs and the perturbation was the weak van der Waals interaction between balls in adjacent tubes.

Particularly striking in this figure is the singular behavior for small $\alpha$, as blown-up in the inset. This behavior is well-known for the lattice-gas, for which the transition temperature satisfies (for weak coupling) [22]

$$
k_{B} T_{c}=\frac{2 J_{l}}{\ln (1 / c)-\ln [\ln (1 / c)]}
$$

Here $J_{l}$ is the longitudinal interaction and $c=J_{t} / J_{l}$ is the anisotropy ratio of transverse to longitudinal interaction strengths. The singular behavior reflects the divergent susceptibility of the 1D system at low $\mathrm{T}$; as the correlation length diverges, larger regions of fluid in adjacent channels are coupled, so increases rapidly with increasing $J_{t}$. The other notable result in this formula and the figure is that the characteristic energy and $T_{c}$ scale are given by the longitudinal coupling, which is about $500 \mathrm{~K}$ for the peapod case, whereas naively one might have expected to be proportional to $J_{t}$. 


\section{Acknowledgments}

We are grateful to DOE and NSF for support of this research and to S. J. Full and N. Wada for help with figures.

[1] S. J. Full, J. P. McNutt, M. W. Cole, M. Mbaye and S. M. Gatica, Anomalous effective dimensionality of quantum gas adsorption near nanopores, to appear in J. Phys.:Cond. Mat.

[2] J. Vavro, M. C. Llaguno, B. C. Satishkumar, D. E. Luzzi, and J. E. Fisher, Appl. Phys. Lett. 80, 1450 (2002); D. E. Luzzi, B. W. Smith, Carbon 38, 1751 (2000); D. J. Hornbaker et al., Science 295, 828 (2002).

[3] M. M. Calbi, S. M. Gatica, and M. W. Cole, Phys. Rev. B67, 205417 (2003).

[4] N. Wada and M. W. Cole, J. Phys. Soc. Japan 77, 111012 (2008).

[5] J. Taniguchi, et al, Phys. Rev. Lett. 94, 065301 (2005).

[6] Y. Matsushita, T. Matsushita, R. Toda, M. Hieda and N. Wada, AIP Conf. Proc. 850, 297 (2006).

[7] V.A. Yurovsky, M. Olshanii, and D. S. Weiss, Adv. Opt At. Mol. Phys. 55, 61 (2007).

[8] M. M. Calbi, S. M. Gatica, M. J. Bojan, G. Stan and M. W. Cole, Rev. Mod. Phys. 73, 857 (2001).

[9] A. C. Dillon and M. J. Heben, Appl. Phys. A Mater. 72, 133 (2001).

[10] M. M. Calbi, M. W. Cole, S. M. Gatica, M. J. Bojan and J. K. Johnson, in Adsorption by Carbons, ed. E. J. Bottani and Juan M. D. Tascón (Elsevier Science Publishing, 2008), pp. 187-210.

[11] J. K. Johnson and M. W. Cole, in Adsorption by Carbons, op cit, pp. 369-402.

[12] S. M. Gatica, M. M. Calbi, R. D. Diehl and M. W. Cole, J. Low Temp. Phys. 152, 89 (2008).

[13] We exclude the case of an infinitely long-range interaction, for which mean-field theory correctly predicts a transition in 1 D. See M. Kac, G. E. Uhlenbeck and P. C. Hemmer, J. Math. Phys. 4, 216 (1963).

[14] A. D. Migone, in Adsorption by Carbons, op cit, Chapter 16.

[15] S. M. Gatica, M. J. Bojan, G. Stan, and M. W. Cole, J. Chem. Phys. 114, 3765 (2001).

[16] M. M. Calbi, S. M. Gatica, M. J. Bojan and M. W, Cole, J. Chem. Phys. 115, 9975 (2001).

[17] R. Toda, M. Hieda, T. Matsushita, N. Wada, J. Taniguchi, H. Ikegami, S. Inagaki and Y. Fukushima, Phys. Rev. Lett. 99, 255301 (2007).

[18] G. Stan, and M. W. Cole, Surf. Sci. 395, 280 (1998).

[19] R. K. Pathria, Statistical Mechanics, second edition (Butterworth-Heinemann, Oxford, 1996), Fig. 6.5.

[20] G. Stan, S. M. Gatica, M. Boninsegni, S. Curtarolo, and M. W. Cole, Am. J. Phys. 67, 1170 (1999).

[21] R. A. Trasca, M. M. Calbi, M. W.Cole, and J. L. Riccardo, Phys. Rev. E69, 011605 (2004).

[22] M. E. Fisher, Phys.Rev. 162, 480 (1967); T. Graim and D. P. Landau, Phys. Rev. B24, 5156 (1981). 